\title{
Determination of number of termite mounds supported by computational vision
}

\author{
$1^{\text {st }}$ Vanessa Ap. de Moraes Weber \\ Universidade Católica Dom Bosco (UCDB) \\ Campo Grande, Brasil \\ Universidade Estadual do MS (UEMS) \\ Dourados, Brasil \\ vamoraes@gmail.com
}

\author{
$2^{\text {nd }}$ Fabricio de Lima Weber \\ Pós Grad. em Zoot. Prod. Animal no Cerrado Pantanal \\ Universidade Estadual do MS (UEMS) \\ Aquidauana, Brasil \\ Faculdade de Computação (FACOM) \\ Universidade Federal do MS (UFMS) \\ Campo Grande, Brasil \\ fabricio.weber@gmail.com
}

\author{
$3^{\text {rd }}$ Edilson Silveira \\ Universidade Católica Dom Bosco (UCDB) \\ Campo Grande, Brasil \\ edilson.silveira@ifms.edu.br
}

\author{
$4^{\text {th }}$ Gilberto Luciano de Oliveira \\ Universidade Católica Dom Bosco (UCDB) \\ Campo Grande, Brasil \\ gilbertolucoli@hotmail.com
}

\author{
$5^{\text {th }}$ Marcelo Folhes \\ Geociência Aplicada \\ IBIGEO \\ City, Country \\ marcelo@ibigeo.com.br
}

\author{
$6^{\text {th }}$ Michel Constantino \\ Universidade Católica Dom Bosco (UCDB) \\ Campo Grande, Brasil \\ michel@ucdb.br
}

\author{
$7^{\text {th }}$ Hemerson Pistori \\ Universidade Católica Dom Bosco (UCDB) \\ Campo Grande, Brasil \\ Faculdade de Computação (FACOM) \\ Universidade Federal do MS (UFMS) \\ Campo Grande, Brasil \\ pistori@ucdb.br
}

\begin{abstract}
Resumo-The determination of the number of termite mounds in a pasture area is a factor that can determine the stage of degradation of the same. The termite count work has been done manually which becomes costly. Our work aims to present a comparison of image filtering techniques and the best time to collect aerial images with UAV (Unmanned Aerial Vehicles) in order to count the number of termite mounds by means of computer vision. Comparisons were made between the number of mounds counted in the image by a specialist with the number of mounds in the image after being submitted to computer vision techniques. It was identified that height is the main variable and that of the five techniques used, Gaussian and Bilateral presented the best results.
\end{abstract}

Index Terms - aerial images, termite mound, unmanned aerial vehicle (UAV)

\section{INTRODUCTION}

O Mato Grosso do Sul é destaque no cenário nacional em relação ao número do rebanho de bovinos, totalizando 22.487 milhões de cabeças em 2017, representando uma participação de $10,14 \%$ do rebanho total do Brasil [1]. Este estado conta com uma das maiores áreas de pastagem do Brasil, as quais, desde os anos 70 passam por transformações de áreas nativas para produtoras de agricultura e pecuária [2]. A criação de gado no MS é essencialmente a pasto ou extensiva e as condições de pastagens são de grande importância para a manutenção dessa atividade. A criação extensiva de bovinos tem sido a atividade econômica mais importante para a região do Pantanal do Mato Grosso do Sul [3].

Assim, [4] afirma que quando se tem uma grande densidade de cupins em uma determinada área eles se alimentam de folhas vivas o que de certa forma favorece a degradação da pastagem. Nesse sentido, as pastagens tornam-se um ambiente propício que facilita a proliferação de cupinzeiros por causa da homogeneidade e da ausência de competidores [5].

Em experimentos anteriores [5] [6] [7] fica evidente que a coleta de cupins e a contagem do número de montículos ocorreu de forma tradicional, na qual separa-se uma área específica, em hectare ou talhões e posteriormente faz-se a contagem a campo um a um. Entretanto com o amparo tecnológico [8] utilizou imagens de satélites disponibilizadas pelo Google Earth e estimou a quantidade de 90 milhões de montículos de cupins numa determinada área no nordeste de Minas Gerais e no semiárido da Bahia, Brasil.

A contagem de cupinzeiros nas regiões de pastagens representa um dado importante para tomada de decisão de pecuaristas, ambientalistas e demais profissionais que utilizam esse dado como referência. A partir dessa premissa, foram analisados trabalhos e pesquisas para identificar a metodologia usada na determinação do número de cupinzeiros e todos os 
trabalhos encontrados se referem a contagem de forma manual no local da pastagem, com exceção de [8] que usou imagens de satélite para tal tarefa.

Segundo as definições de [9], o uso de análise de imagens e recursos tecnológicos como a coleta de imagens por VANTs (Veículos Áereos não Tripulados) e seu processamento por softwares apoiados em visão computacional são indicadores de uma pecuária tecnificada e de precisão. Apoiados na definição de pecuária de precisão foram coletadas imagens áreas, com VANTs, de pastagens na região do Pantanal Sul Mato Grossense e analisadas por um software desenvolvido pelo Inovisão, que é um grupo de pesquisa criado em 2004 na Universidade Católica Dom Bosco (UCDB), cujo objetivo principal é a integração da pesquisa, do desenvolvimento e da inovação para contribuir para o desenvolvimento do estado de Mato Grosso do Sul.

Veículos aéreos não tripulados é uma forma relativamente barata de adquirir imagens e vídeos e tem sido muito usado principalmente para gerar ou apoiar decisões na agricultura e na pecuária. A versatilidade do VANTs permite que o usuário adapte diferentes tipos de dispositivos de captura de imagens, o que facilita configurar o aparelho de acordo com a necessidade e aplicação do mesmo [10].

A visão computacional vem sendo utilizada no intuito de se extrair dados a partir de imagens. Dessa forma, manipula-se esses dados para que os mesmos sejam utilizados nos mais diversos propósitos [11]. Assim, a visão computacional é uma área da computação que visa a criação de programas de computador capazes de extrair de forma automática ou ao menos semiautomática informações relevantes contidas em imagens digitais para resolver ou auxiliar na solução de problemas que dependam direta ou indiretamente dessas imagens. As principais funções típicas encontradas em sistemas de visão computacional são aquisição de imagem, pré-processamento, extração de características, detecção e segmentação, e processamentos de alto nível.

$\mathrm{O}$ uso de VANTs associados à sistemas de visão computacional para análise de imagens com aplicação na pecuária foi abordado por alguns autores. Dentre eles, [12] usaram algoritmos de redes neurais convolucionais para contagem e monitoramento de bovinos. Numa revisão sobre o uso de VANT, aplicado a sensoriamento remoto e áreas vegetais [13] cita que na África do Sul e na Austrália tem sido usado essa ferramenta para cuidados com animais.

\section{TRABALhos Relatados}

Nas pastagens do estado de Mato Grosso do Sul, destacase infestações de cupins do gênero Cornitermes, dividindo-se em três tipos mais comuns $C$. cumulans, $C$. bequaerti e $C$. silvestrii [14]. Uma grande infestação de cupinzeiros podem ser considerados pragas em pastagens, haja vista que os mesmos colaboram para acelerar a degradação da área utilizada para o trato do rebanho, sendo que os cupins consomem folhas vivas. Algumas espécies de cupins podem ser úteis, pois ao construírem túneis no solo podem acelerar o processo de recuperação do solo e reposição de minerais [15]. No Brasil podem ser encontradas mais de 290 espécies de cupins, mas somente 10 a $20 \%$ tem relevância na economia.

Com relação a contagem de montículos de cupins, em um levantamento feito por [16], a média de cupinzeiros por hectare no Centro Oeste brasileiro era de 76,84. Sendo a espécie Cornitermes com maior incidência nos cupinzeiros com $77 \%$ de todas as espécies. A importância de poder determinar a infestação dos cupins de montículos pode ajudar não só na pecuária, como também lavouras ou plantações de eucaliptos e pinus [17]. Contudo, compreender a organização destes indivíduos, bem como as suas áreas de maiores infestações, poderá determinar a melhor maneira de controle e prevenção destas espécies.

Os cupins devem muito do seu sucesso evolutivo e ecológico a sua capacidade digestiva, isso porque estão entre um dos poucos organismos que conseguem digerir celulose, um dos compostos biológicos mais abundantes da terra e que é mais comum de se encontrar em madeiras [18]. Na América do Sul existem cerca de 400 espécies registradas de cupins. Na área agrícola esses insetos prejudicam principalmente as culturas de cana de açúcar, arroz da montanha e eucalipto. Com relação a pastagens existem controvérsias e não se pode afirmar que os cupins realmente prejudicam as pastagens [19] [20].

Contudo, o objeto de estudo mais abordado dentre os pesquisadores é o seu habitat, ou seja, sua moradia denominada de montículo e também conhecida popularmente como cupinzeiro. A complexidade, a engenharia que existe por trás do projeto arquitetônico do cupinzeiro, intriga e ao mesmo tempo é enaltecida pelos pesquisadores ao longo do globo. Os cupins são considerados mestres em construção entre os insetos, eles aplicam nas mais diversas construções um composto de argila, saliva e fezes [18].

A resistência e altura dos montículos são comparadas e até mesmo, copiada pela humanidade [21]. Outros aspectos importantes são a durabilidade e a vitalidade do cupinzeiro. A descrição de cupinzeiros abandonados na República Popular do Congo onde o maior tinha idade entre 2119 e 2335 anos e o menor com idade entre 684 e 796 anos [22].

Para [23] e [24] a presença de ninhos de cupins (cupinzeiros) está associada à degradação da pastagem, no entanto [6] afirma que não é evidente que a diminuição da fertilidade, o aumento da acidez do solo ou até mesmo o aparecimento dos ninhos seja consequência da degradação ou que seja, um fenômeno natural induzido pela falta de controle dos mesmos. Embora os estudos e pesquisas sobre os cupins estejam em evidência, existe uma carência no que se refere à abundância, distribuição espacial e diversidade dessas espécies existentes no cerrado [7]. Contudo, a evolução tecnológica amparada por métodos cada vez mais eficazes de pesquisas, tem favorecido novas descobertas sobre a vida dos cupins. Assim, com essa nova perspectiva, imagem de satélites disponibilizada pelo Google Earth, à medida que tiveram uma melhor resolução, favoreceu para que cupinzeiros muito grandes no nordeste brasileiro fossem evidenciadas devido aos seus notórios contornos [8].

Em áreas de pastagens, no que se refere à infestação de 
cupins existem várias discussões, sobre o benefício [16] ou malefício do inseto já que esse ambiente é propício e facilita a proliferação de cupinzeiros por causa da homogeneidade e da ausência de competidores [5]. Segundo [16] os cupins preferem fixar sua residência em áreas mais altas do terreno. $\mathrm{O}$ inseto produz montículos nas pastagens que prejudicam a utilização de maquinários na agricultura, reduz a área útil do solo e que também servem de abrigos para animais indesejáveis como cobras, escorpiões e aranhas [20].

No Mato Grosso do Sul, [25] relata que apenas $0,5 \mathrm{~m}^{2}$ é a área de ocupação média de cada cupinzeiro, o que não justificaria dizer que os cupins reduzam de forma significante a área utilizada como pastagens. Em concordância, [16] consideram importante a necessidade de se conhecer melhor os cupins de montículos, uma vez que não se tem verificado danos causados por esses animais. $\mathrm{O}$ autor acima citado ainda relata que esses insetos, do ponto de vista da fertilidade do solo, são úteis para a renovação dos nutrientes. Segundo [5], não é só a redução da área útil de pastagem que representa um problema causado pela infestação de cupins. Segundo outros autores, quando a espécie se alimenta de gramíneas, sementes e raízes há o risco de diminuição da pastagem para o gado [26]. Adicionalmente, [27] e [24] definem a presença de ninhos de cupins nas pastagens como um fator que determina o grau de degradação e [14] relata que a falta de manutenção na pastagem predetermina o grau de infestação.

A presente pesquisa objetivou investigar e identificar a altura ideal para captura de imagens de cupinzeiros com um VANT, além de definir dentre as técnicas de suavização aplicadas quais são as melhores para contagem de montículos de cupins.

\section{MAterial e MÉtodos}

Para o procedimento inicial da pesquisa foram coletadas imagens de uma área com infestação de cupinzeiros utilizando o VANT. Para tanto, o VANT iniciou sobrevoo vertical sem mudança de trajetória, a partir de um ponto fixo prédeterminado, coletando assim, imagens com intervalo de 5 metros até a altura máxima de 100 metros. Foram coletadas 100 imagens, entretanto somente 22 foram utilizadas para o treinamento do software. As imagens a partir de 45 metros foram descartadas por se tratar de uma região arenosa conforme pode ser observado na imagem da Fig. 2 disponibilizada pelo GoogleEarth ${ }^{\circledR}$. Visualmente percebe-se que a qualidade da imagem se torna prejudicada a partir dessa altura, e até mesmo a visão humana não consegue distinguir com clareza o que é cupinzeiro e o que é a areia do terreno. Dessa forma foram descartadas 78 imagens pois estas tinham sua qualidade prejudicada devido a semelhança da cor do cupinzeiro com a cor da areia do terreno. Na Fig.1 é possível observar exemplos de imagens utilizadas para o treinamento do software enquanto que na Fig. 2 é possível observar o local da coleta das imagens.

O voo sobre a chácara Pérola do Pantanal no município de Rio Verde de MS, no Pantanal, coordenadas geográficas, $18^{\circ} 73^{\prime} 36^{\prime \prime}$ latitude sul, $55^{\circ} 10^{\prime} 45^{\prime \prime}$ longitude Oeste, ocorreu no dia 10 de junho de 2017. Por coletar fotos únicas por altura específica, não precisou gerar mosaicos. Não foram

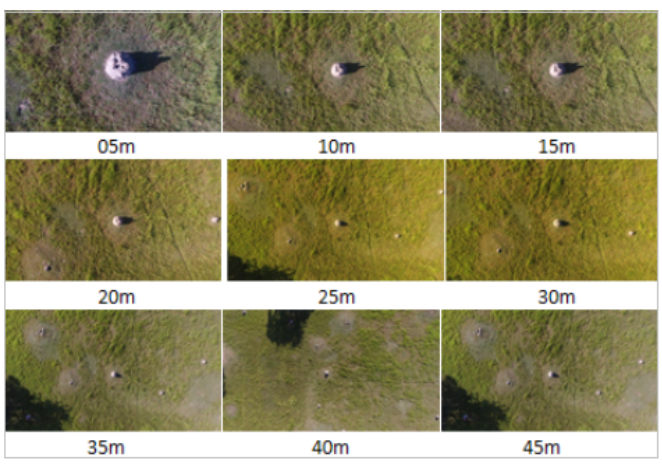

Figura 1. Exemplos de imagens utilizadas no experimento.

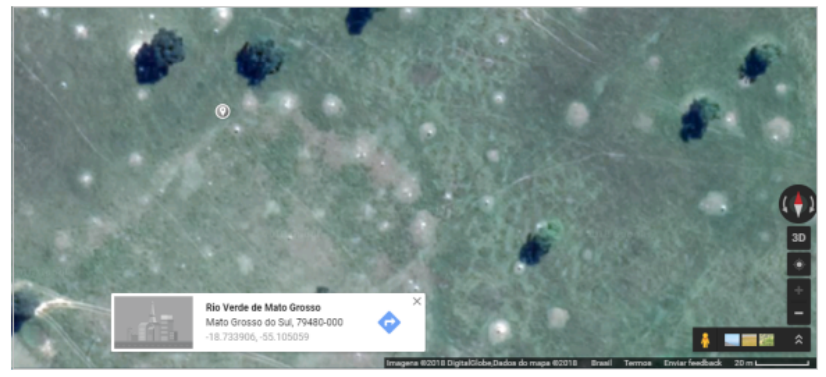

Figura 2. Local da coleta de imagens.

extraídas medidas de cada objeto da imagem e também não foi realizados experimentos envolvendo distâncias, por isso não foi necessário ortorretificar (corrigir a imagem). Para conversão da imagem original em tons de cinza, foi utilizada a ferramenta Conta Cupim, desenvolvida no laboratório de pesquisa do Grupo Inovisão, - Universidade Católica Dom Bosco (UCDB) em Campo Grande - MS.

Inicialmente a ferramenta converteu a imagem original em uma imagem com tons em cinza, posteriormente aplicou-se binarização com limiar fixo, determinando visualmente a partir de uma das imagens. Após esta etapa aplicou-se morfologia matemática através de uma sequência de dilatações e erosões com um núcleo de tamanho de 3 x 3 na imagem com diferentes iterações (número de repetições). Posteriormente, o sistema executa a contagem dos montículos encontrados na imagem mostrando na a imagem original em tons de cinza, a imagem com o filtro aplicado conforme citados a seguir, a imagem com limiar simples binário e a imagem final segmentada juntamente com o número de montículos encontrados, conforme Figura 3.

As seguintes técnicas de filtragem foram aplicadas no experimento:

Passa-Baixa - Calculado pela média de todos os pixels na área do núcleo e substituindo o elemento central pela média encontrada. Determinamos a altura e largura do núcleo em $5 \times 5$.

Gaussiana - Utiliza-se do kernel gaussiano no lugar do filtro de caixa. Com a configuração de largura e a altura do núcleo de $5 \times 5$. Esse filtro é muito eficiente na remoção de ruídos da imagem. 
Mediana - Calcula a mediana para todos os pixels sob a janela do núcleo e o pixel mais ao centro é substituído pelo valor da mediana. Também utilizado para remoção de ruídos. Diferente do filtro da gaussiana o valor mediano sempre será um valor encontrado na imagem.

Bilateral - No Filtro Bilateral também é usado o filtro de gaussiana, mas ao contrário da gaussiana preserva a borda desfocando somente os filtros vizinhos com intensidade diferente da central.

Linear - Trata-se de um filtro de média que permite o processamento linear de uma imagem, utilizando um núcleo de filtro de média 5X5. Assim os pixels que se encontram nesta janela são imperpolados. Aplicando em todos os pixels até que sai uma imagem filtrada.

A contagem dos montículos é realizada através do reconhecimento de objetos restantes, através da sua área em pixel, após aplicação do filtro selecionado, caminhando por estes objetos através de um laço visitando todas as posições x e y (largura e altura) da imagem, no final da contagem a ferramenta salva uma imagem com o resultado obtido conforme Fig. 3.

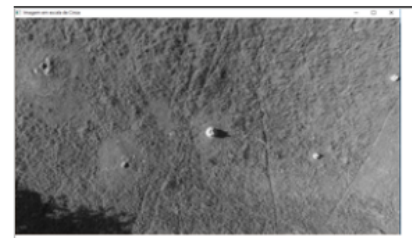

(a)

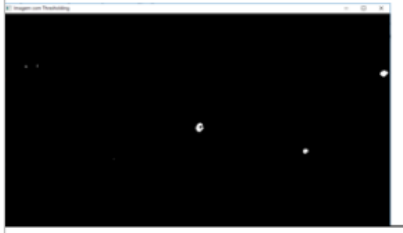

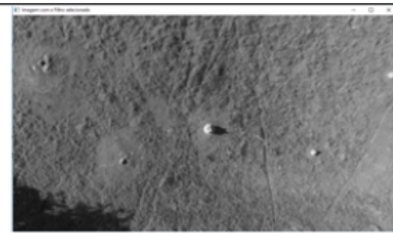

(b)

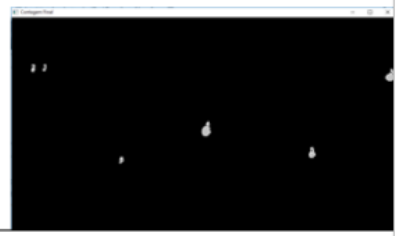

Figura 3. Resultado da Contagem passo a passo: a) Imagem em Tons de Cinza, b) Imagem com o Filtro aplicado, c) Imagem com Thesholding e d) Imagem com Montículos Contados.

$\mathrm{Na}$ Tabela I é possível verificar, que foram encontrados 6 objetos em uma imagem capturada pelo Vant, totalizando uma área total de montículos de 1235 pixels da área total da imagem que era de 275800 pixels.

Tabela I

RESUlTADOS DA CONTAGEM NA ALTURA.

\begin{tabular}{|c|c|}
\hline Número de Objetos & Área em Pixel \\
\hline 1 & 45 \\
2 & 50 \\
3 & 382 \\
4 & 505 \\
5 & 226 \\
6 & 7 \\
\hline Total de Pixel & $\mathbf{1 2 3 5}$ \\
\hline
\end{tabular}

As imagens foram disponibilizadas em um software anotador de imagens desenvolvido pelo grupo Inovisão, que permitiu à um especialista analisar cada imagem, registrar o número de montículos encontrados conforme Fig. 4.

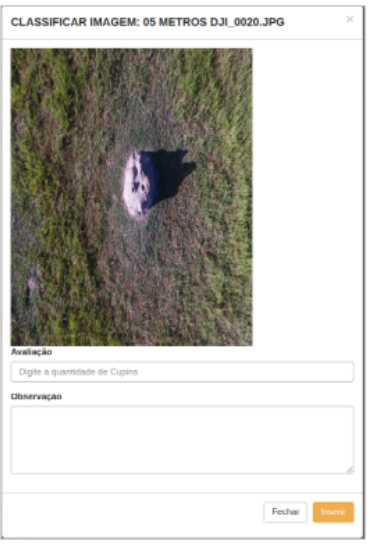

Figura 4. Software anotador de imagens.

Após o processamento da imagem pelo software descrito acima, gerou-se um modelo de regressão Tobit [28], com o objetivo de identificar quais variáveis mais influenciam na determinação de número de montículos de cupins. Para gerar a Regressão Tobit utilizou-se o software R versão 3.3.0. Segundo [29], o modelo de regressão Tobit deve ser utilizado nos casos em que a variável dependente está concentrada em algum dos pontos limites, ou limitada entre faixas de valor.

Nesses casos, a aplicação deste modelo pode contornar o problema por meio de estatísticas que possibilitam fazer inferências para toda a população. As estimativas nesse modelo são obtidas pelo Método de Máxima Verossimilhança. $\mathrm{O}$ modelo de regressão Tobit apresentado nesta pesquisa possui como variável dependente a contagem de cupins pelo especialista considerado o valor verdadeiro do número de cupinzeiros naquela área, e as variáveis independentes deste modelo são: a altura do VANT para a captura das imagens, e os tratamentos aplicados que são: passa-baixa, gaussiana, mediana, bilateral e linear.

\section{RESUlTADOS}

Na Tabela II, Fig. 5 e Fig. 6, estão apresentados os resultados obtidos do modelo Tobit. Analisando o resultado verificase que as variáveis correspondentes a Altura ( $\mathrm{p}$-valor $<2 \mathrm{e}^{-16}$ ), filtro de Gaussiana (p-valor $1.89 \mathrm{e}^{-6}$ ) e o Filtro Bilateral ( $\mathrm{p}$ valor $1.72 \mathrm{e}^{-6}$ ) foram as únicas estatisticamente significantes, com p-valor menor ou igual a $10 \%$.

Os resultados permitem considerar que a altura do VANT na hora de coletar as imagens, é um fator importante para determinar o número de cupinzeiros na contagem por visão computacional e que os filtros Gaussiana e o Bilateral apresentam probabilidade positiva, ou seja, eficiência, devendo ser considerados para o pré-processamento de imagens com o intuito de implementação de um software de contagem automática de cupinzeiros.

A Fig. 5 representa, em um eixo 3D, os resultados contados do filtro Gaussiana com relação à altura do VANT e ao valor 
Tabela II

RESULTADOS OBTIDOS NA REGRESSÃO TOBIT.

\begin{tabular}{|c|c|}
\hline Métrica & p-valor \\
\hline altura & $<2 \mathrm{e}^{-16 * * *}$ \\
linear & 0.815 \\
passa baixa & 0.522 \\
gaussiana & $1.89 \mathrm{e}^{-6}$ \\
mediana & 0.490 \\
bilateral & $1.72 \mathrm{e}^{-6}$ \\
\hline
\end{tabular}

contado pelo especialista. Os valores apresentam uma correlação positiva, valores extremos e corrobora com o resultado significativo do modelo Tobit.

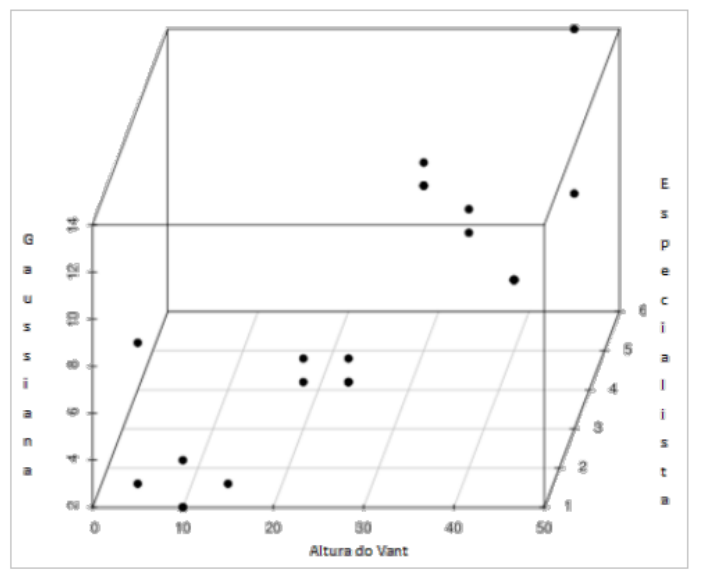

Figura 5. Representação da relação entre altura do VANT, filtro da Gaussiana e a contagem feita pelo especialista.

Na Fig. 6 abaixo apresenta os dados do Filtro Bilateral com relação à altura do VANT e a variável de avaliação do especialista apresenta menor correlação, porém, evidências de correlação positiva com dados concentrados em pontos específicos.

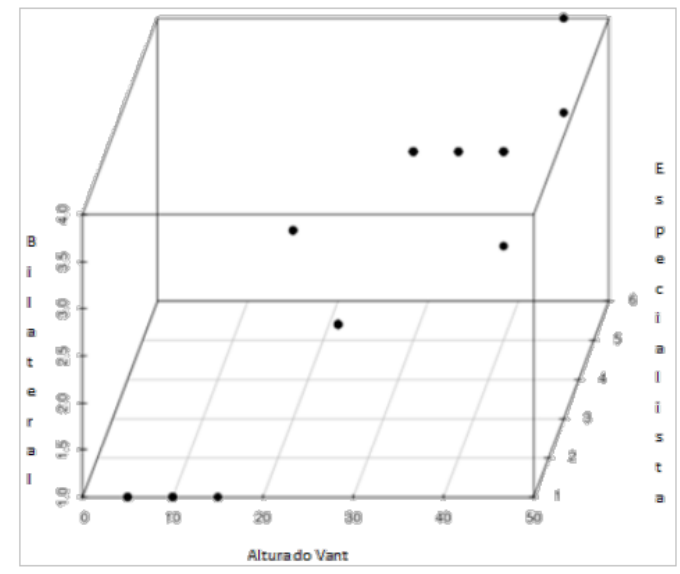

Figura 6. Representação da relação entre a altura do VANT, o filtro bilateral e a contagem feita pelo especialista.

Foi realizado o teste Anova para verificar o desempenho das técnicas utilizadas pelo software Conta Cupim. Os resultados demonstram que as técnicas utilizadas pelo software para contagem dos cupins não obtiverão resultados estatisticamente significantes conforme podem ser observados na Tabela III, pois o p-valor foi de 0.33804. Entretanto, a altura do VANT na hora da captura da imagem tem significância estatística na contagem de montículos de cupins, em conformidade com o p-valor de 0.000378 conforme apresentado na Tabela III.

Tabela III

RESULTADOS OBTIDOS NA REGRESSÃO TOBIT.

\begin{tabular}{|c|c|}
\hline Variável & $\boldsymbol{p}$-valor \\
\hline Técnica aplicada & 0.33804 \\
Altura do Vant & $\mathbf{0 . 0 0 0 3 7 8}$ \\
\hline
\end{tabular}

\section{CONCLUSÃo}

A pecuária de precisão apresenta tecnologias para melhorar o desempenho das propriedades rurais, testar métodos que avance na tomada de decisão e redução de custos são fundamentais para melhorar a produtividade. O objetivo da presente pesquisa foi de comparar técnicas de suavização de imagens, com intuito de melhorar a contagem de montículos de cupim em áreas degradadas.

$\mathrm{O}$ resultado apontou que os filtros Gaussiana e o Bilateral apresentam eficiência, devendo ser considerados para o préprocessamento de imagens com o intuito de implementação de um software de contagem automática de cupinzeiros. Em trabalhos futuros sugere-se que sejam testadas imagens adquiridas com o uso de câmaras multiespectrais a fim de medir a vegetação nas redondezas dos montículos. Sugere-se também implementar e avaliar outras técnicas de segmentação e identificação de visão computacional, apoiadas por inteligência artificial como superpixel ou segmentação por meio de redes neurais convolucionais para que se possa melhorar a detecção e contagem de cupinzeiros nas pastagens. A principal contribuição deste trabalho foi de utilizar uma forma inovadora para a contagem de montículos de cupins.

\section{AGRADECIMENTOS}

Este trabalho foi realizado com o apoio financeiro da Coordenação de Aperfeiçoamento de Pessoal de Nível Superior Brasil (CAPES) - Código de Financiamento 001, Conselho Nacional de Desenvolvimento Científico e Tecnológico (CNPq), Fundação de Apoio ao Desenvolvimento da Educação, Ciência e Tecnologia do Estado do Mato Grosso do Sul (FUNDECT) e Universidade Católica Dom Bosco (UCDB).

\section{REFERÊNCIAS}

[1] ABIEC - Associação Brasileira das Indústrias Exportadoras de Carne. Perfil da pecuária no Brasil, Relatório Anual. Acesso em <16/09/2018>. Disponível em <http://abiec.siteoficial.ws/images/upload/sumario-pt010217.pdf>.

[2] Kluthcouski, J.; Aidar, H.; Stone, L.F.; Cobucci, T. 2004. Integração lavoura-pecuária e o manejo de plantas daninhas. Informações Agronômicas, n.106, p.1-20

[3] EMBRAPA - Empresa Brasileira de Pesquisa Agropecuária. Levantamentos aéreos de espécies introduzidas no Pantanal: porcos ferais (porco monteiro), gado bovino e búfalos. Acesso em <20/11/2017> Disponível em $<$ https://www.infoteca.cnptia.embrapa.br/bitstream/doc/810746/1/BP28.pdf> 
[4] Fontes, L. R. 1998. Cupins nas pastagens do Brasil: algumas indicações de Controle. In: FEALQ (Ed.). Cupins: o desafio do conhecimento. Piracicaba: FEALQ, p. 211-225.

[5] Cunha, H. F. H; P. P. A. M. Morais, 2010. Relação espécie-área em cupinzeiros de pastagem, Goiânia-GO, Brasil. EntomoBrasilis, 3: 6063.

[6] Lima, S. S. S.; Alves, B. J. R.; Aquino, A. M.; Mercante, F. M.; Pinheiro, E. F. M.; Sant'anna S. A. C.; et al. 2011. Relação entre a presença de cupinzeiros e a degradação de pastagens. Pesquisa Agropecuária Brasileira; 46(12): 1699-1706.

[7] Peres Filho, O.; Souza, J.C.; Souza, M. D.; Dorval, A. 2012. Distribuição espacial de cupinzeiros de Cornitermes snyderi (Isoptera: Termitidae) e sua associação com teca. Pesquisa Florestal Brasileira; 32(70): 59-66.

[8] Funch, R. R. 2015. Termite mounds as dominant land forms in semiarid northeastern Brazil. Journal of Arid Environments, v. 122, p. 27-29.

[9] Cáceres, E. N.; Pistori, H.; Turine, M. A. S.; Pires,P. P.; Soares.C.O.; Carromeu, C. 2011. Computational Livestock Precision - Position Paper In: Second Workshop of the Brazilian Institute for Web Science Research, 2-3 Agosto, Rio de Janeiro.

[10] Pistori, H.; Da Silva, G. 2016. As múltiplas aplicações dos DRONES na agricultura. A Granja, v. 806.

[11] Rios, L. R. S. 2010. Visão computacional. Departamento de Ciência da computação - Universidade Federal da Bahia (UFBA) Salvador, Bahia, Brasil.

[12] Charmoso, P.; Raveane, W; Parra, V., Gonzaléz, A. 2014. UAVs applied to the counting and monitoring of animals. In: Ramos C, Novais P, Nihan CE, Corchado Rodríguez JM (eds) Ambient intelligence-software and applications, vol 291. Advances in intelligent systems and computing. Springer, New York, pp 71-80.

[13] Salamí, E.; Barrado, C.; Pastor, E. 2014. UAV flight experiments applied to the remote sensing of vegetated areas. "Remote Sensing", 11 Novembre, vol. 6, núm. 11, p. 11051-11081.

[14] Valério, J. R. 2006. Cupins-de-montículo em pastagens. Campo Grande: Embrapa Gado de Corte, p. 33. (Embrapa Gado de Corte. Documentos, 160).

[15] Miklós, A. A. de W. 1998. Papel de cupins e formigas na organização e na dinâmica da cobertura pedológica. In: FONTES, L. R.; BERTI-FILHO, E. (Ed.). Cupins: o desafio do conhecimento. Piracicaba:FEALQ, p. 227-241.

[16] Czepak, C; De Araújo, E. A.; Fernandes, P. M. 2007. Ocorrência de espécies de cupins de montículo em pastagens no Estado de Goiás. Pesquisa Agropecuária Tropical (Agricultural Research in the Tropics), v. 33 , n. 1 , p. $35-38$.

[17] ABRAF - Associação Brasileira De Produtores de Florestas Plantadas. Anuário estatístico ABRAF 2013 ano base 2012 / ABRAF. - Brasília: 2013.

[18] Oberst, S.; Lai, J. C S; Evans, T. A. 2016. Termites utilise clay to build structural supports and so increase foraging resources. Scientific reports, v. 6.

[19] Constantino, R. 1998. Catalog of the termites of the New World (Insecta: Isoptera). Arquivos de Zoologia (São Paulo) 35(2):135-231.

[20] Constantino, R. 2002. The pest termites of South America: taxonomy, distribution and status. Journal of Applied Entomology, v. 126, n. 7-8, p. 355-365.

[21] Vasconcelos C. A. 2000. Livro Estruturas da natureza: um estudo entre a interface e a biologia, Studio Nobel.

[22] Erens, H. Et Al. 2015. The age of large termite mounds-radiocarbon dating of Macrotermes falciger mounds of the Miombo woodland of Katanga, DR Congo. Palaeogeography, Palaeoclimatology, Palaeoecology, v. 435 , p. $265-271$.

[23] Macedo, N. 1995. Atualização no controle de cupins subterrâneo em cana-de-açúcar. In: BERTI FILHO, E.; FONTES, L.R.(Ed) Alguns aspectos atuais da biologia e controle de cupins. Piracicaba, FEALQ, p.121-126

[24] Aquino, A. M.; Correia, M. E. F.; Alves, M. V. 2008. Diversidade da macrofauna edáfica no Brasil. In: MOREIRA, F.M.S.; SIQUEIRA, J. O.; BRUSSAARD, L. (Ed.). (Org.). Diversidade do solo em ecossistemas brasileiros. Lavras: UFLA, p.143-170.

[25] Valério, J. R. 1995. Ocorrência, danos e controle de cupins de montículo em pastagens p. 33-36. In Reunião Sul Brasileira de Insetos de Solo, 5. Dourados, MS. Ata e Resumos. 110p.

[26] Fernandes, P. M.; Czepak, C.; Veloso, V. R. S. 1998. Cupins de montículos em pastagens: prejuízo real ou praga estética?, p. 187-210. In Cupins: o desafio do conhecimento. FEALQ, Piracicaba, SP. 512 p.
[27] Santos, R. S. M. Dos; Oliveira, I. P. De; Morais, R. F. De; Urquiaga, S.C.; Boddey, R. M.; Alves, B. J. R. 2007. Componentes da parte aérea e raízes de pastagens de Brachiaria spp. em diferentes idades após a reforma, como indicadores de produtividade em ambiente de Cerrado. Pesquisa Agropecuária Tropical, v.37, p.119-124.

[28] Tobin, J. 1958. Estimation of relationships for limited dependent variables. Econometrica: journal of the Econometric Society, p. 24-36.

[29] Greene, W. H. 2008. Econometric Analysis. 6th edition. Prentice Hall. 